\title{
MRI EVALUATION OF NORMAL PITUITARY GLAND WITH AGE AND SEX RELATED VARIATIONS IN KASHMIRI POPULATION
}

\author{
AKSHIT KUMAR ${ }^{1}$ \\ Department of Radio Diagnosis, Sher E Kashmir Institute of Medical Sciences, Soura, Srinagar, India
}

\begin{abstract}
To study the size and mean volume of normal pituitary gland in different age groups of both genders with Magnetic Resonance Imaging (MRI). The patients were divided into six groups according to age. Mean height of pituitary gland was greater in female patients than that of male patients in the same age group except in 1-10 year age group. Height of the pituitary gland reached its maximum in the 21 to 30 years of age group in both sexes, after which, there was a decline in the pituitary height in the subsequent age groups till 50 years. The overall mean pituitary height in the age group 1-10 years came out to be $5.6 \pm 1.6 \mathrm{~mm}$. In the age group 11-20 years, mean pituitary height was $6.3 \pm 1.8 \mathrm{~mm}$. In the age group 21-30 years $6.8 \pm 1.9 \mathrm{~mm}, 31-40$ years age group $6.4 \pm 1.1 \mathrm{~mm}$, in $41-50$ years age group $6.2 \pm 1.3 \mathrm{~mm}$ and individuals above 50 years of age, mean pituitary height was observed as $6.3 \pm 1.9 \mathrm{~mm}$. the most common shape was flat in all age groups and both sexes which was seen in $58 \%$ of people followed by convex in $28 \%$ and concave shape in $14 \%$ patients. The mean pituitary volume in the age group 1-10 years came out to be males $216 \pm 15.6$ $\mathrm{mm} 3$ and in females $204 \pm 16.5 \mathrm{~mm}$. In the age group 11-20 years, mean pituitary volume in males was $348 \pm 58 \mathrm{~mm} 3$ and in female's 305 $\pm 116 \mathrm{~mm} 3$. In the age group 21-30 years, mean pituitary volume in males was $417 \pm 78 \mathrm{~mm} 3$ and in females $452 \pm 78$ mm3. In the age group 31-40 years, mean pituitary volume in males was 376 $\pm 51 \mathrm{~mm} 3$ and in females was $426 \pm 44 \mathrm{~mm}$. In $41-50$ age groups mean pituitary volume in males was $342 \pm 76 \mathrm{~mm} 3$ and in females it was $410 \pm 56 \mathrm{~mm} 3$. In individuals above 50 years of age, the mean pituitary volume was observed in males as $421 \pm 158 \mathrm{~mm}$ and $443 \pm 68 \mathrm{~mm} 3 \mathrm{in}$ females. A complete knowledge of physiological variation in the size and shape of pituitary gland is necessary to assess the abnormalities involving the pituitary gland. In cases with a borderline abnormality in the size of pituitary gland, further evaluation by dynamic contrast study on MRI should be done.Pituitary size and shape evaluation needs baseline values with the normal range of height and volume of the normal pituitary gland in various age groups in both genders in Kashmiri population.
\end{abstract}

KEYWORDS: Magnetic Resonance Imaging (MRI), pituitary gland, Kashmiri

The pituitary gland is located in sella turcica within the sphenoid bone. This region has marked variability amongst normal individuals, including pneumatisation of sphenoid sinus, size of sella turcica and size and shape of pituitary gland (Chaudhary and Bano, 2011) (Elster, 1993).

A systematic approach to the pathologies of pituitary region is very important because sometimes findings are very subtle.In majority of the patients ,normal can be easily differentiated from abnormal, however, a significant grey zone exists likephysiological hypertrophy of gland, subtle microadenoma, increased lobulated margins, inflammatory diseases and the reporting radiologist faces a dilemma whether the pituitary is within the normal limits or is abnormal.Measurements of the normal pituitary gland for various age ranges are helpful to diagnose such casesHence, for complete assessment of pituitary gland, one should be aware of its normal anatomy as well as physiological variations in its size and shape in different age groups in both males and females. Changes are seen in size as well as shape of pituitary gland which happen due to changes in complex hormonal physiology of the gland. MRI has proved to be an accurate diagnostic modality for the assessment of pituitary gland. Despite its importance, only a few studies have been performed to analyze the size, shape and structure of the normal pituitary gland. The majority of the known published data is in excess of 20 years ago and these studies were specific to certain populations such as pediatrics (Doraiswamy et al., 1992) (Hayakawa et al., 1989) (Suzuki et al., 1990) (Wiener et al., 1985). Necessity of the study arises from the lack of previous instances of measurement of pituitary height and volume in such a wide variety of age groups and for both sexes in a designated Kashmiri population. Therefore, the present study was conducted to study the size and shape of normal pituitary gland in different age groups of both genders with MRI and to evaluate mean normal volume of the pituitary gland in relation with age and gender.

\section{MATERIALS AND METHODS}

This was a prospective observational study done in department of Radiodiagnosis at a tertiary care institute.

${ }^{1}$ Corresponding author 
Institutional ethics committee clearance was obtained for the study. The study period extended from July 2018 to April 2019 in which 350 patients were included in the study (136 males and 214 females, ranging from one year up to 92 years of age) who underwent brain MRI in the Radiodiagnosis department for variety of reasons like suspected metastases, meningitis, seizures etc, however, patients who were suffering from significant endocrine disorder; head injury, genetic syndrome, pregnant or breast feeding or patients having empty sella with pituitary gland having height of less than $2 \mathrm{~mm}$ were not included in this study and subsequent analysis. The patients were subsequently divided into 6 age groups ( 1 to 10 years, 11 to 20 years, 21 to 30 years, 31 to 40 years, 41 to 50 years, 50 years above) for both genders.

MRI examinations were performed using Siemen's Mangnetom Avanto 1.5 Tesla scanner. The various measurements were taken on sagittal T1 weighted images and coronal T1 weighted images. MRI protocol for sagittal scan was matrix 320x240, FOV was $230 \mathrm{~mm}$ and $5 \mathrm{~mm}$ slice thickness were taken and for coronal scan matrix was 320x196, FOV was $230 \mathrm{~mm}$ with $5 \mathrm{~mm}$ slice thickness.

The mid-sagittal image $\mathrm{T} 1$ weighted image was used to obtain all pituitary gland height (cranio-caudal) and Anterior-Posterior (AP) dimensions (Figure 1a). Pituitary gland width (transverse) was measured on a coronal $\mathrm{T} 1$ weighted slice through the pituitary stalk (Figure 1b). Pituitary gland volume was estimated by using the formula: $\mathrm{V}=$ Antero-posterior dimension $\mathrm{X}$ Craniocaudal dimension $\mathrm{X}$ Transverse dimension X 0.52 . Pituitary gland shape was obtained in the mid-sagittal T1WI and was observed as flat, convex or concave.

Mean and Standard deviations of pituitary height were calculated in the scale of $\mathrm{mm}$. Volume in different age groups were calculated in the scale of $\mathrm{mm} 3$. Relation between mean height and age and relation between volume and age were done by ANOVA test, Chi-square test and pvalue $<0.05$ was considered as significant.
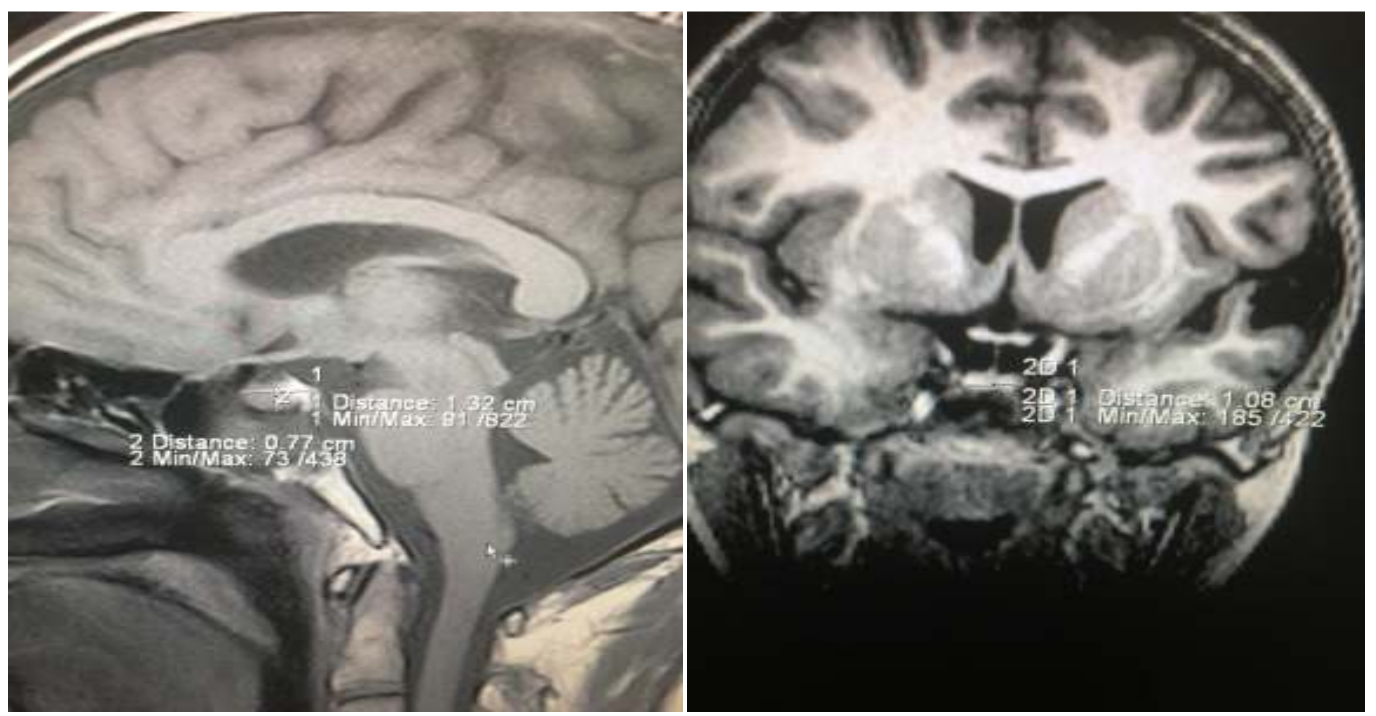

Figure 1a \& 1b: Midline sagittal and coronal T1 weighted images showing height AP dimension and transverse dimension of pituitary gland in a 52 year old female with height of $7.7 \mathrm{~mm}$

\section{RESULTS}

The mean pituitary height in our study group was $6.3 \pm 1.8 \mathrm{~mm}$. The mean pituitary height in the age group 110 years came out to be $5.6 \pm 1.6 \mathrm{~mm}$. In the age group 1120 years, mean pituitary height was $6.3 \pm 1.8 \mathrm{~mm}$, in the age group 21-30 years $6.8 \pm 1.9 \mathrm{~mm}, 31-40$ years age group $6.4 \pm 1.1 \mathrm{~mm}$, in $41-50$ years age group $6.2 \pm 1.3 \mathrm{~mm}$ and individuals above 50 years of age mean pituitary height was observed as $6.3 \pm 1.9 \mathrm{~mm}$ (Table 1). Relation of pituitary height with age came out to be statistically insignificant ( $p$ value 0.17 ) in between the male age groups, however, pvalue was 0.002 which was statistically significant in between the females age groups. The mean value of pituitary height and volume in different age group and in both the sexes were given in the Table 2 . 
The difference in pituitary volume was statistically significant in between the groups for both male and female age groups $(\mathrm{p}=0.002)$. Consequently, the mean height of pituitary gland in female patients of each age group was greater than that of male patients in the same age group except in the age group of 1-10 years and 11-20 years. However, representative differences in the mean pituitary heights were observed only in the 21-30 years old age group, 31-40-year-old age group and the 50-year-old and above age group. The height of pituitary gland in both males and females was less in the 1-10 years age group (Table 2), increasing in puberty and reached maximum in the 21-30 years age group, and declined in 30-50 years and above age groups, except in the 50 years above age group in women, in which again there was an increment in the mean pituitary height (Figure 2a and $2 b$ ).

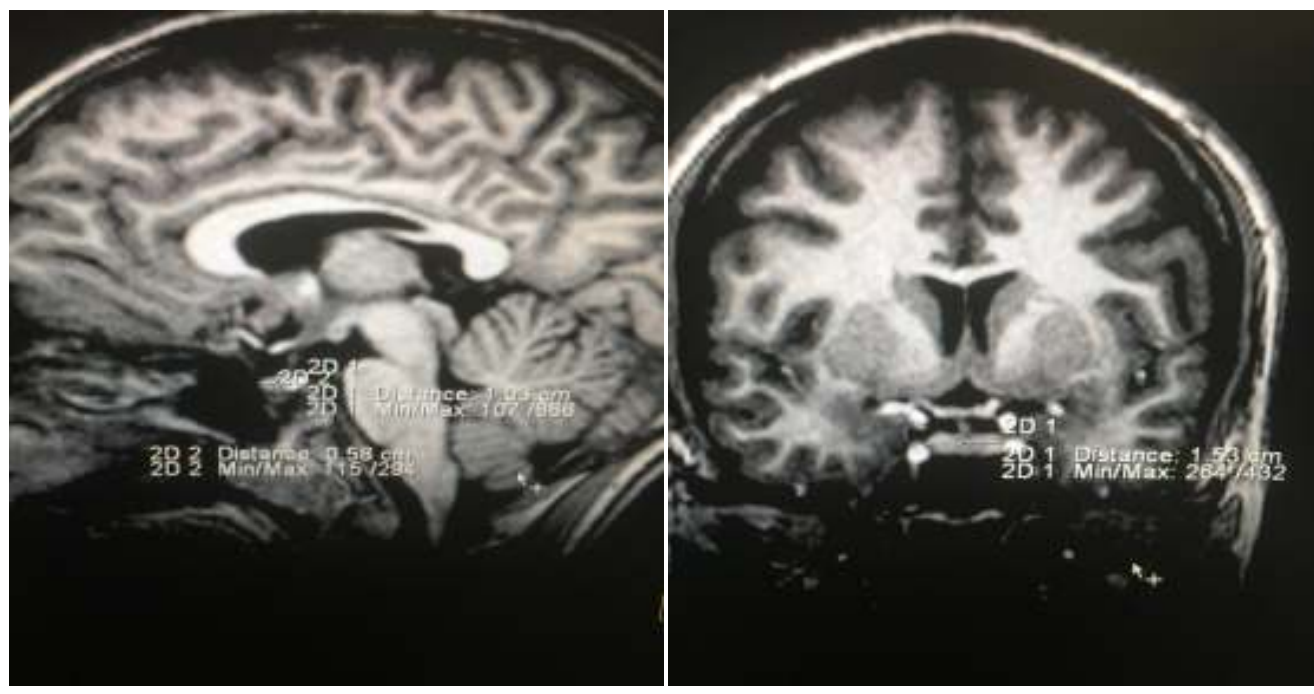

Figure 2a \& 2b: Midline sagittal and coronal T1 weighted images showing height AP dimension and transverse dimension of pituitary gland in a 3 year old male child with height of $5.8 \mathrm{~mm}$

The most common shape was flat which was seen in $58 \%$ of people, convex shape was seen in $28 \%$ (Figure $3 \mathrm{a}$

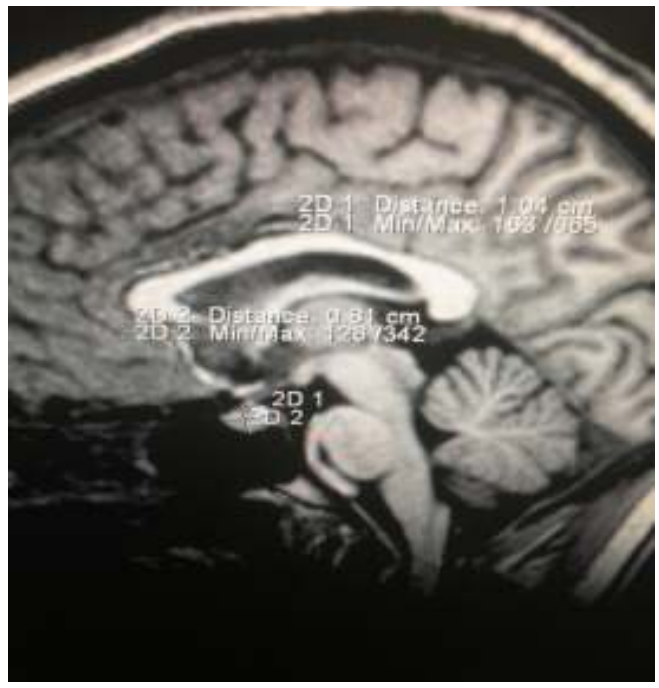
and $3 b)$ and concave shape was seen in $14 \%$ in all age groups and both sexes.

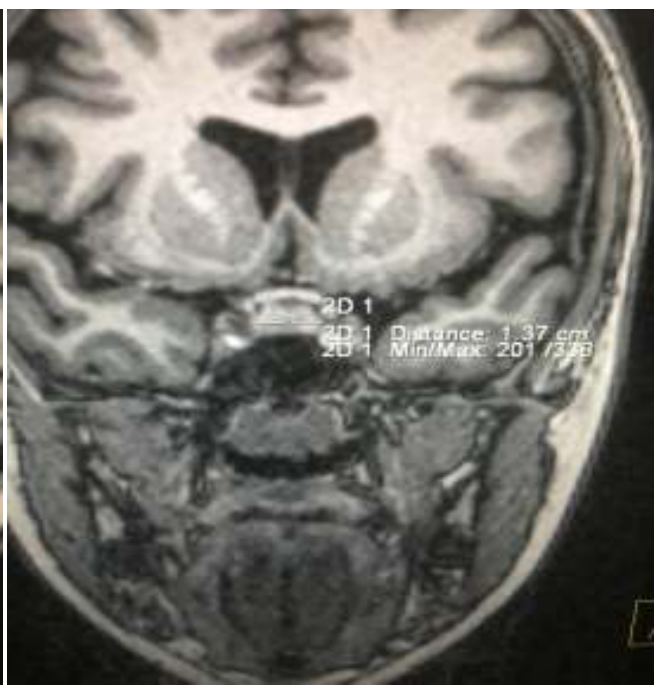

Figure 3a \& 3b: Midline sagittal and coronal T1 weighted images showing various dimensions of pituitary gland in a 25 year old female with height of $8.1 \mathrm{~mm}$ with convex upper border 
Table 1: Mean height and volume of pituitary gland in different age groups

\begin{tabular}{|c|c|c|}
\hline Age Group (years) & Mean height in $\mathbf{~ m m}( \pm \mathbf{S D})$ & Mean volume in $\mathbf{~ m m 3}( \pm$ SD) \\
\hline $1-10$ & $5.6( \pm 1.6)$ & $208( \pm 20.5)$ \\
\hline $11-20$ & $6.3( \pm 1.8)$ & $323( \pm 72)$ \\
\hline $21-30$ & $6.8( \pm 1.9)$ & $438( \pm 70)$ \\
\hline $31-40$ & $6.4( \pm 1.1)$ & $402( \pm 61)$ \\
\hline $41-50$ & $6.2( \pm 1.3)$ & $387( \pm 48)$ \\
\hline$>50$ & $6.3( \pm 1.9)$ & $438( \pm 61)$ \\
\hline
\end{tabular}

Table 2: Mean height and pituitary volume in different age groups with respect to sex

\begin{tabular}{|c|c|c|c|}
\hline Age group(years) & Sex & Mean height in mm (SD) & Mean volume in mm3(SD) \\
\hline \multirow{2}{*}{$1-10$} & Male & $5.9( \pm 1.5)$ & $216( \pm 15.6)$ \\
\cline { 2 - 4 } & Female & $5.4( \pm 1.8)$ & $204( \pm 16.5)$ \\
\hline \multirow{2}{*}{$11-20$} & Male & $6.4( \pm 1.8)$ & $348( \pm 58)$ \\
\cline { 2 - 4 } & Female & $6.1( \pm 2.0)$ & $305( \pm 116)$ \\
\hline \multirow{2}{*}{$21-30$} & Male & $6.3( \pm 1.1)$ & $417( \pm 78)$ \\
\cline { 2 - 4 } & Female & $7.1( \pm 1.2)$ & $452( \pm 78)$ \\
\hline \multirow{2}{*}{$31-40$} & Male & $6.1( \pm 1.4)$ & $376( \pm 51)$ \\
\cline { 2 - 4 } & Female & $6.6( \pm 1.2)$ & $426( \pm 44)$ \\
\hline \multirow{2}{*}{$41-50$} & Male & $6.0( \pm 1.4)$ & $342( \pm 76)$ \\
\cline { 2 - 4 } & Female & $6.3( \pm 1.7)$ & $410( \pm 56)$ \\
\hline \multirow{2}{*}{$>50$} & Male & $6.0( \pm 1.3)$ & $421( \pm 58)$ \\
\cline { 2 - 4 } & Female & $6.5( \pm 1.3)$ & $443( \pm 68)$ \\
\hline
\end{tabular}

\section{DISCUSSION}

Of all the results compounded during our study, the most interesting result was that the height of the pituitary gland in females increases again in the 50-59-yearold age group. Increase in pituitary height during puberty is well known (Doraiswamy et al., 1992) (Elster et al., 1990), and decrease in height of pituitary gland with age is also documented in many studies (Doraiswamy et al., 1992) (Suzuki et al., 1990) (Elster et al., 1990) (Peyster et al., 1983). Studies suggest that it is the changes in the hormone levels which cause such changes in the pituitary morphology. The increase in pituitary height during puberty can be related to the increased production of Luteinizing Hormone (LH) during this time of growth. The decrease in the pituitary height with age also shows the changes in the endocrine status with aging and also physiological atrophy of the pituitary gland.

It has been found that the basal serum concentrations of gonadotropic hormones (LH, FSH) decreased after puberty up to the fifth decade of life ( $\mathrm{Ju}$ et al., 2010). However, the concentrations of these hormones again rise in females in fifth and sixth decades, because of age-related decrease in circulating gonadal steroids hormones (estrogens and progesterone) and an increase in gonadotropin releasing hormones due to decreased feedback effect (Ju et al., 2010). As per our study, females have a greater pituitary height in the same age group (i.e., 50 years and above). Previous studies (Doraiswamy et al., 1992) have also shown the same result possibly due to compensatory hypertrophy after a significant decrease in gonadal steroid feedback effect.

The height of pituitary gland increases with age in younger people has been documented previously (Doraiswamy et al., 1992) (Suzuki et al., 1990) (Argyropoulou et al., 1991) (Elster et al., 1990) (Peyster et al., 1986). Studies have also reported that height of pituitary gland reaches its maximum in the 10-19-year-old age group of both sexes (Suzuki et al., 1990) (Argyropoulou et al., 1991) (Elster et al., 1990) or in the 20-29 years old age 


\section{KUMAR: MRI EVALUATION OF NORMAL PITUITARY GLAND WITH AGE AND SEX RELATED VARIATIONS...}

group (Hayakawa et al., 1989), findings consistent with our study.

Sanjay et al., (2014) concluded that mean height of pituitary gland was $6.27 \pm 0.56$. They also observed that the height decreases as the age advances with a mild increase in height in 40-49 years age group.

The changes in pituitary size are basically due to changes in pituitary gland height, as there are no age-related effects on gland length or width. As suggested by Lurie et al., (1990) future studies might reasonably use pituitary height alone with findings on mid-sagittal T1-weighted images serving as the single measure of pituitary size. As seen in our study, the pituitary gland is often concave in its superior aspect, and this would not be reflected in a single, thick sagittal image, which may show only the higher lateral margins of the gland. This can cause errors in the measurements of the pituitary gland height. This can lead to decreased gland even though the pituitary height is increased (as it was measured from the lateral margins of the gland). The same problem is also seen in partially empty sellae where we can see the gland tissue extending up to the full height along its lateral margins which may lead to error in the measurements.

The results of our study were based on evaluation of pituitary size for 350 patients. As per the results of this study, height of pituitary gland reached a maximum in the 20 to 29 years of age group in both males and females, after which there was a decline in the pituitary height in the subsequent age groups.

A pituitary height greater than $9 \mathrm{~mm}$ in the 20 to 29 age group or $8 \mathrm{~mm}$ in the other age groups should be considered abnormal. In the females, the pituitary height showed a significant increase again in the 50 to 59 years age group which is due to the hypertrophy after a greater postmenopausal loss of gonadal steroid feedback mechanism. This data should help in the further evaluation of pituitary morphology in various neuro-endocrinal disorders.

Our study did provide a data on the normal pituitary height and volume in the Kashmiri population in different age groups. Normal pituitary gland dimensions were influenced by age and gender. Pituitary height peaked in 21-30 years age group. Pituitary height more than $9 \mathrm{~mm}$ in 21-30 years age group and more than $8 \mathrm{~mm}$ in other age group was considered as abnormal. Perimenopausal women show increase in pituitary height again which might be due to loss of gonadal steroid feedback. This observation in the normal variation of pituitary size and shape is helpful in evaluation of pituitary morphology in neuroendocrine disorders.

\section{CONCLUSION}

This study provides up-to-date data regarding the normal dimensions of the pituitary gland. The study gives normal data regarding the pituitary gland with regards to sex and age of different patients.Pituitary size can be accurately assessed by using MRI and can be correlated with the patient's age and sex. In the cases where there is a borderline abnormality in the size and shape of pituitary gland, it should be further evaluated by dynamic contrast study on MRI. This study provides the normal range of height and volume of the normal pituitary gland in various age groups in both genders in Kashmiri population.

\section{REFERENCES}

Argyropoulou M., Perignon F., Brunelle F., Brauner R. and Rappaport R., 1991. Height of normal pituitary gland as a function of age evaluated by magnetic resonance imaging in children. Pediatr Radiol., 21(4):247-49.

Chaudhary V. and Bano S., 2011. Imaging of the pituitary: Recent advances. Indian J. Endocrinol Metab., 15(Suppl3): S216-S223.

Doraiswamy P.M., Potts J.M., Axelson D.A., Husain M.M., Lurie S.N. and Na C., 1992. MR assessment of pituitary gland morphology in healthy volunteers: age- and gender-related differences. AJNR Am. J. Neuroradiol, 13(5):1295-99.

Elster A.D., 1993. Imaging of the sella: anatomy and pathology. Semin Ultrasound CT MR, 14(3): 182194.

Elster A.D., Chen M.Y.M., Williams D.W. and Key L.L., 1990. Pituitary gland: MR imaging of physiologic hypertrophy in adolescence. Radiology., 174(3 Pt 1):681-85.

Hayakawa K., Konishi Y., Matsuda T., Kuriyama M., Konishi K. and Yamashita K., 1989. Development 
KUMAR: MRI EVALUATION OF NORMAL PITUITARY GLAND WITH AGE AND SEX RELATED VARIATIONS...

and aging of brain midline structures: assessment with MR imaging. Radiology. 172(1):171-77.

Ju K., Bae H.G., Park H., Chang J., Choi S. and Sim K., 2010. Morphometric study of (10)the Korean adult pituitary glands and the diaphragma sellae. J Korean Neurosurg Soc., 47(1):42-47.

Lurie S.N., Doraiswamy P.M., Husain M.M., Boyko O.B., Ellinwood E.H. Jr. and Figiel G.S., 1990. In vivo assessment of pituitary gland volume with magnetic resonance imaging: the effect of age. J. Clin Endocrinol Metab., 71(2):505-08.

Peyster R.G., Adler L.P., Viscarello R.R., Hoover E.D. and Skarznski J., 1986. CT of the normal (8)pituitary gland. Neuroradiology, 28(2):161-65.

Peyster R.G., Hoover E.D., Viscarello R.R., Moshang T. and Haskin M.E., 1983. CT appearance (9)of the adolescent and preadolescent pituitary gland. AJNR Am. J. Neuroradiol., 4(3):411-14.

Sanjay S.C., Subbaramaiah M. and Jagannatha S.R., 2014. Variation in size and shape of a (1)normal adult female pituitary gland: a radiological study. Journal of Evolution of Medical and Dental Sciences, 3(18):4934-39.

Suzuki M., Takashima T., Kadoya M., Konishi H., Kameyama T. and Yoshikawa J., 1990. Height of normal pituitary gland on MR imaging: age and sex differentiation. J. Comput. Assist. Tomogr., 14(1):36-39.

Wiener S.N., Rzeszotarski M.S., Droege R.T., Pearlstein A.E. and Scafron M., 1985. Measurement of pituitary gland height with MR imaging. AJNR Am. J. Neuroradiol., 6(5):717-22. 